\title{
Comparison of techniques for antimicrobial susceptibility testing of mycobacteria
}

\author{
D I Limb, P F Wheat, R C Spencer, G S Harris, A B Rayner, B Watt
}

\begin{abstract}
Aims-To evaluate adenosine triphosphate (ATP) bioluminescence as a rapid technique for antimicrobial susceptibility testing of Mycobacterium spp by comparing it with conventional and radiometric methods, and to assess its potential for use in clinical microbiology laboratories.

Methods-115 clinical isolates from a wide range of mycobacterial species and four control organisms of known susceptibility were tested against six antimicrobial agents. Minimum inhibitory concentrations (MICs) were determined after 4-6 weeks' incubation on Middlebrook 7 H10 agar. Susceptibility was also determined radiometrically using a Bactec 460, and by bioluminescent assay of ATP using a 1250 luminometer (LKBWallac).
\end{abstract}

Results-Susceptibility results after 7 days showed excellent correlation with conventionally determined MICs. 714 susceptibility tests were performed by both techniques, with seven major discrepancies between the two systems. For pyrazinamide, agreement was $100 \%$, but five strains of $M$ tuberculosis, including one control, and 11 mycobacteria other than $M$ tuberculosis (MOTT) failed to grow on Middlebrook agar at pH 5·5. 606 tests were performed by radiometry, with four major discrepancies between this technique and ATP bioluminescence.

No particular species of Mycobacterium gave aberrant results. Contamination was a problem; 12 of the 119 strains tested were contaminated at day 1 and had to be repeated before results were obtained. Contamination of individual tests increased significantly after 7 days of incubation.

Department of Bacteriology, Royal Hallamshire Hospital, Glossop Road, Sheffield S10 2JF

D I Limb

P F Wheat

R C Spencer

Scottish Mycobacteria Reference Laboratory, City Hospital,

Edinburgh

G S Harris

A B Rayner

B Watt

Correspondence to:

Dr Limb

Accepted for publication 21 October 1992 Conventional methods for antimicrobial sus based on growth on solid agar and require a minimum of three to four weeks of incubation. ${ }^{12}$ The resistance ratio method using Lowenstein-Jensen (LJ) medium is most commonly used in the United Kingdom and the rest of Europe, ${ }^{3}$ while the proportional and absolute concentration methods on Middlebrook agar are favoured in the United States. ${ }^{4}$ During this prolonged incubation, patients with resistant organisms might receive inappropriate treatment that could result in the infecting organism becoming resistant to additional drugs. Furthermore, the incidence of infections caused by mycobacteria other than $M$ tuberculosis (MOTT) has increased sharply with the emergence of AIDS..$^{5-7}$ It has been estimated that up to $50 \%$ of patients with AIDS develop mycobacterial infection at some stage of their disease, ${ }^{8}$ and many of these isolates show aberrant drug resistance. Rapid determination of resistance may therefore reduce the need for ineffective and highly toxic drugs.

Several techniques for the rapid susceptibility testing of mycobacteria have been described. Radiometric methods for cultivating and for antimicrobial susceptibility testing have been in use in diagnostic microbiology for more than a decade. In 1975 Cummings and colleagues used $\left[{ }^{14} \mathrm{C}\right]$ glycerol and $\left[{ }^{14} \mathrm{C}\right]$ acetate as substrates for mycobacterial growth. ${ }^{9}$ Middlebrook et al further developed the technique and introduced a medium containing $\left[{ }^{14} \mathrm{C}\right]$ palmitic acid as the labelled substrate. ${ }^{10}$ It was the work of Kertcher et al, however, who first demonstrated the possibility of using radiometry as a rapid susceptibility method for mycobacteria. ${ }^{11}$ Nowadays an increasing number of laboratories use this technique. Correlation with conventional methods is good and results are often available in four to seven days. ${ }^{12-14}$

The firefly bioluminescent ATP assay has long been recognised as an accepted method for estimation of microbial biomass. ${ }^{15}$ The assay involves the measurement of light produced as a result of an enzymic reaction catalysed by the firefly Photinus pyralis (American firefly). The amount of light produced is directly proportional to the amount of ATP involved in the reaction. The technique is based on several assumptions which experimental evidence has shown to be correct: all living organisms contain ATP; ATP is not associated with dead cells; ATP can be extracted from microbial cultures and accurately measured.

ATP bioluminescent techniques have been 
of various micro-organisms including mycobacteria. ${ }^{16-19}$ Nilsson et al found full agreement in $99 \%$ of assays with the resistance ratio method on $\mathrm{LJ}$ medium, and $98 \%$ were in full agreement with the radiometric system. ${ }^{16}$

\section{Methods}

One hundred and fifteen distinct clinical isolates were tested: $M$ malmoense $\mathrm{n}=29 ; M$ tuberculosis $\mathrm{n}=26 ; M$ avium-intracellulare $\mathrm{n}=$ 25; $M$ xenopi $\mathrm{n}=12 ; M$ kansasii $\mathrm{n}=8 ; M$ fortuitum $\mathrm{n}=6 ; M$ gordonae $\mathrm{n}=4 ; M$ bovis $\mathrm{n}=$ 2; $M$ chelonei $\mathrm{n}=2 ; M$ scrofulaceum $\mathrm{n}=1$. Four control strains H37Rv and C3105 (M tuberculosis), $\mathrm{C} 10795$ ( $M$ bovis), and $\mathrm{C} 10268$ ( $M$ kansasii) were also tested. These were kindly supplied by the Scottish Mycobacteria Reference Laboratory, Edinburgh, the Royal Brompton National Heart and Lung Hospital, London, and the Mycobacteria Reference Unit, Cardiff. These were stored at $-70^{\circ} \mathrm{C}$ in $10 \%$ glycerol broth until required, then subcultured on to Middlebrook $7 \mathrm{H} 10$ agar (BBL) three weeks before testing.

Streptomycin (Sigma), ethambutol (Lederle), rifampicin (Merrell), pyrazinamide (Sigma), ciprofloxacin (Bayer) and isoniazid (Roche) were made up as standard solutions according to manufacturers' instructions and filter sterilised before each experiment.

Conventional susceptibility testing was performed using the absolute concentration method using Middlebrook $7 \mathrm{H} 10$ agar. The medium was prepared according to the manufacturer's instructions, and OADC supplement (BBL) was added and distributed in $2.75 \mathrm{ml}$ amounts into sterile bijoux bottles containing $0.25 \mathrm{ml}$ of antimicrobial agent. For pyrazinamide, the $\mathrm{pH}$ of the medium was adjusted to $5 \cdot 5$. Each bijou was then sloped and allowed to set. The range of concentrations was $0 \cdot 125$ to $32 \mathrm{mg} / \mathrm{l}$ except for pyrazinamide, which was 16 to $256 \mathrm{mg} / \mathrm{l}$. Drug free slopes were also prepared for use as controls.

From a three week culture of the mycobacterial strain, colonies were emulsified in $3 \mathrm{ml}$ of Middlebrook 7H9 broth (BBL) containing

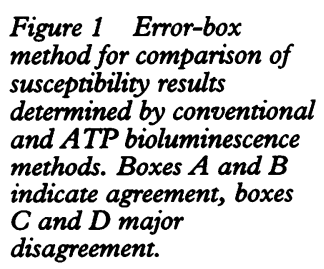

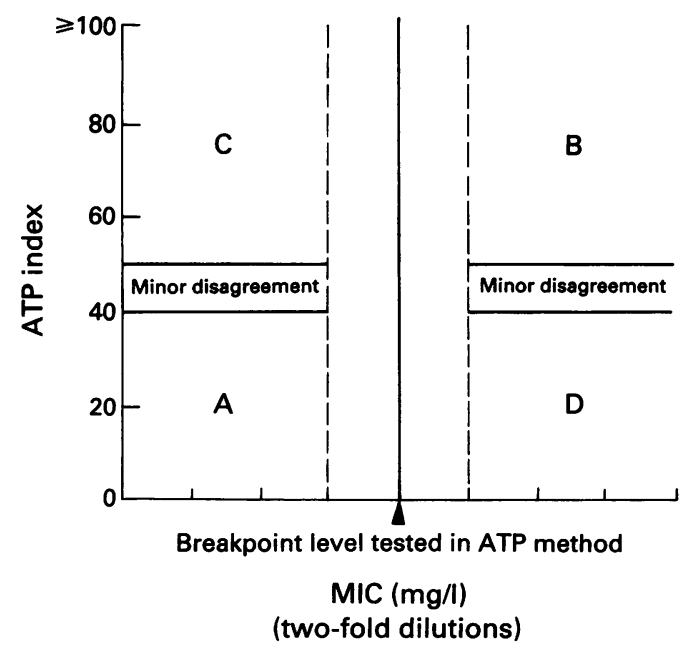

$0.05 \%$ Tween 80 and several sterile glass beads. Each sample was homogenised for 2 minutes in a class 1 biological safety cabinet using a Spinmix (Gallenkamp), and large particles were allowed to settle. The supernatant fluid was removed and adjusted to McFarland 0.5 standard (about $10^{6}$ mycobacteria/ml) using sterile $7 \mathrm{H} 9$ broth so that a $1 \mu \mathrm{l}$ inoculum gave about 1000 colony forming units. To prevent the inoculum running to the bottom of the container the bijou was positioned horizontally overnight. The slopes were then incubated aerobically at $35^{\circ} \mathrm{C}$ for up to six weeks and examined regularly. The minimum inhibitory concentration (MIC) was defined as the lowest concentration of antimicrobial agent that reduced growth of the test organism to $1 \%$ or less when compared with the control slope ${ }^{32}-10$ colonies or less.

For susceptibility testing by ATP bioluminescence, antimicrobial agents were prepared in $2.5 \mathrm{ml}$ volumes in Middlebrook $7 \mathrm{H} 9$ broth supplemented with OADC enrichment and $0.05 \%$ Tween 80 . For pyrazinamide, the $\mathrm{pH}$ was adjusted to $6 \cdot 0$. The following concentrations were tested: streptomycin $4 \mathrm{mg} / \mathrm{l}$; isoniazid $0.25 \mathrm{mg} / 1$; ethambutol $2 \mathrm{mg} / \mathrm{l}$; rifampicin $0.5 \mathrm{mg} / \mathrm{l}$; pyrazinamide $100 \mathrm{mg} / 1$ and ciprofloxacin $2 \mathrm{mg} / \mathrm{l}$. Colonies of mycobacteria were suspended in $3 \mathrm{ml} 7 \mathrm{H} 9$ broth plus three glass beads, homogenised for 2 minutes, and large particles allowed to settle. The supernatant fluid was adjusted to McFarland 0.5 standard (about $10^{6}$ mycobacteria $/ \mathrm{ml}$ ), and $0.5 \mathrm{ml}$ was added to the tubes containing antimicrobial agents. Control broths of each strain without antimicrobial agent were also included. These were then incubated aerobically at $35^{\circ} \mathrm{C}$ for up to 10 days. Purity was checked by subculture on to blood agar and Middlebrook $7 \mathrm{H} 10$ agar.

In a class 1 biological safety cabinet, a $100 \mu \mathrm{l}$ sample from the mycobacterial culture was pipetted into a glass tube containing $400 \mu \mathrm{l}$ of boiling $0 \cdot 1 \mathrm{M}$ TRIS buffer (BDH) (pH 7.75) plus $2 \mathrm{mmol} / \mathrm{l}$ EDTA (Sigma). After 5 minutes the extract was allowed to cool to room temperature. ATP monitoring reagent $(50 \mu \mathrm{l})$ (LKB-Wallac) was added to the extract and the light intensity recorded using a 1250 luminometer (LKB-Wallac). ATP assays were performed at days $0,1,3$, 5, 7 and 10 of incubation. Results were expressed as an ATP index:

ATP index $=\frac{\text { Relative light units in test }}{\text { Relative light units in control }} \times 100$

Results were analysed by the modified error-box method described by Wheat et $a^{1819}$ (fig 1). Strains in boxes A and B were categorised as showing agreement, strains in boxes $\mathrm{C}$ and $\mathrm{D}$ as showing major disagreement, and strains with ATP indices of $41-49 \%$ as showing minor disagreement.

For radiometric susceptibility testing, antimicrobial agents were prepared in sterile distilled water and $0.1 \mathrm{ml}$ added to a Bactec 12B phial (Becton-Dickinson) ( $\mathrm{pH} \mathrm{6.8)} \mathrm{to}$ give the following final concentrations; strep- 

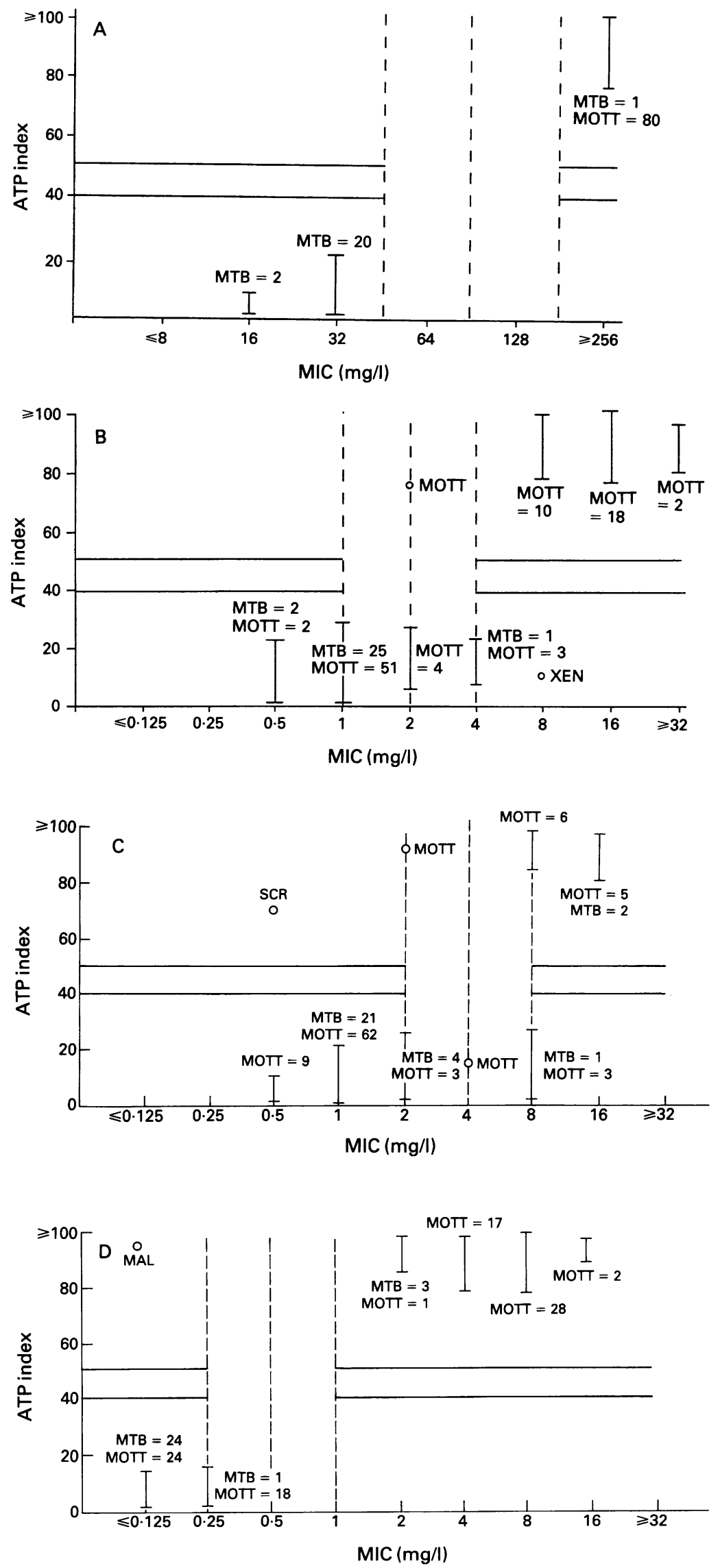

Figure 2 Error-box analyses comparing conventional MIC values with ATP bioluminescence for $M$ tuberculosis (MTB) and non-M tuberculosis (MOTT) strains after seven days of incubation in the presence of $(A)$ pyrazinamide $100 \mathrm{mg} / \mathrm{l}$, (B) ethambutol $2 \mathrm{mg} / \mathrm{l}$, (C) streptomycin $4 \mathrm{mg} / \mathrm{l}$, (D) rifampicin $0.5 \mathrm{mg} / \mathrm{l}$. Discrepant results are highlighted using the species code below. TUB, M tuberculosis; $M A L, M$ malmoense; GOR, M gordonae; SCR, M scrofulaceum; MAI, M avium-intracellulare; FOR, $M$ fortuitum; KAN, $M$ kansasii; $C H E L, M$ chelonei; $X E N, M$ xenopi. I indicates the range of $A T P$ indices at a given MIC value. tomycin $4 \mathrm{mg} / 1$; isoniazid $0.1 \mathrm{mg} / \mathrm{l}$; ethambutol $2 \mathrm{mg} / \mathrm{l}$; rifampicin $0.5 \mathrm{mg} / \mathrm{l}$; ciprofloxacin $2 \mathrm{mg} / \mathrm{l}$. For pyrazinamide, PZA medium (Becton-Dickinson) was used. The final concentration of the drug was $100 \mathrm{mg} / \mathrm{l}$ at a $\mathrm{pH}$ of $6 \cdot 0$.

In a biological safety cabinet mycobacterial colonies were emulsified in $0.5 \mathrm{ml}$ diluent in a sterile bijou bottle, the suspension agitated, and $0.1 \mathrm{ml}$ of this added to a $12 \mathrm{~B}$ phial which was then incubated at $37^{\circ} \mathrm{C}$. The phial was read daily on a Bactec 460 (BectonDickinson) until a suitable growth index (GI) was obtained. This was then used to inoculate the phials containing antimicrobial agents $(0.1 \mathrm{ml})$ to give a final inoculum of about $10^{5}$ mycobacteria $/ \mathrm{ml}$. Phials were incubated for up to 10 days and the GI monitored daily. When the GI reading was 30 or more, the results were interpreted by calculating the change in GI from the previous day. ${ }^{1221}$ If the daily increase in GI in the control was greater than that in the phial containing drugs the organism was reported as susceptible. If the increase was less than that in the phial the organism was reported as being resistant.

\section{Results}

Susceptibility results obtained by bioluminescence after seven days showed excellent correlation with conventionally determined MICs (figs 2A-F). Seven hundred and fourteen susceptibility tests were performed by both techniques, with seven major discrepancies between the two systems (table). Five of these discrepant results showed susceptibility by bioluminescence but resistance by the conventional method. For pyrazinamide, agreement was $100 \%$, but five strains of $M$ tuberculosis, including one control and 11 strains of MOTT, failed to grow on Middlebrook agar at $\mathrm{pH}$ 5.5. Increasing the $\mathrm{pH}$ to 6.0 improved growth but increased MIC values by up to 4 -fold.

Six hundred and six tests were performed by radiometry, with four major discrepancies between this technique and ATP bioluminescence (fig 3). These were for streptomycin (n $=2)$ and ethambutol $(n=1)$ where the result was "susceptible" by radiometry but "resistant" by bioluminescence. A further strain was "resistant" to ethambutol by bioluminescence but "susceptible" by radiometry. There was only one occasion when both of these methods showed disagreement (resistance) with the conventional method (susceptible), but this was due to poor growth of the organism on Middlebrook agar. On repetition of this strain agreement was reached after incubation was extended to six weeks. No particular species of Mycobacterium gave aberrant results. Contamination was a problem; 12 of the 119 strains tested had to be repeated before results were obtained at seven days.

\section{Discussion}

Conventional methods for susceptibility testing of mycobacteria are widely used in clinical 

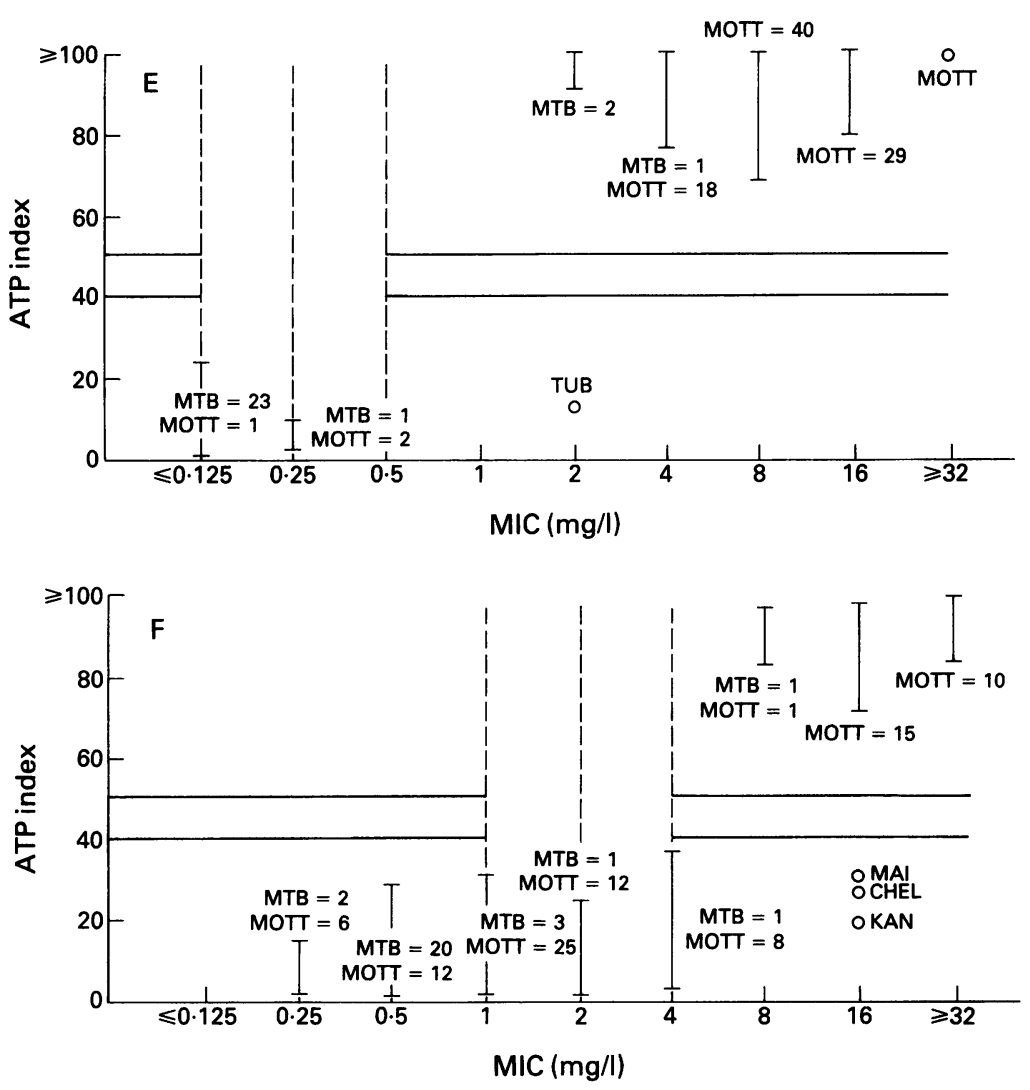

microbiology. The main disadvantage is that they require a prolonged incubation period, usually three to four weeks, which reduces the value of the result to the clinician. This study confirms the work of Nilsson et al ${ }^{16}$ and Beckers et al $^{17}$ who showed that growth of mycobacteria in fluid media may be monitored by ATP bioluminescence, and that the technique lends itself to rapid antimicrobial susceptibility testing. Both of these studies showed excellent correlation with conventional techniques, with results available in four to seven days. We found that seven days was more practical, using the ATP method, and that correlation with the conventional method was $99 \%$. The time needed to obtain reliable results also depends on the size of the inoculum. Nilsson et al showed that when a starting inoculum of less than $10^{4}$ mycobacteria/ml is used for susceptibility testing by the ATP assay, the proportion of resistant bacteria required to demonstrate resistance may not be detected. ${ }^{16}$ Inocula of $10^{6}$ mycobacteria $/ \mathrm{ml}$ or greater, however, may contain increased numbers of resistant variants that will

Summary of discrepancies between susceptibility results (714 tests representing 119 strains of mycobacteria) obtained by ATP bioluminescence and conventional MIC determination

\begin{tabular}{lllll}
\hline Organism & $\begin{array}{l}\text { Antimicrobial } \\
\text { agent }\end{array}$ & $\begin{array}{l}\text { MIC } \\
(\mathrm{mg} / 1)\end{array}$ & $\begin{array}{l}\text { ATP } \\
\text { index }\end{array}$ & $\begin{array}{l}\text { Interpretation } \\
\text { of } A T P \text { assay }\end{array}$ \\
\hline M avium-intracellulare & Ciprofloxacin & 16 & 31 & Sensitive \\
$M$ chelonei & Ciprofloxacin & 16 & 31 & Sensitive \\
$M$ kansasii & Ciprofloxacin & 16 & 20 & Sensitive \\
$M$ malmoense & Rifampicin & $0 \cdot 125$ & 94 & Resistant \\
M scrofulaceum & Streptomycin & $0 \cdot 5$ & 70 & Resistant \\
M tuberculosis & Isoniazid & 2 & 13 & Sensitive \\
$M$ xenopi & Ethambutol & 8 & 13 & Sensitive \\
\hline
\end{tabular}

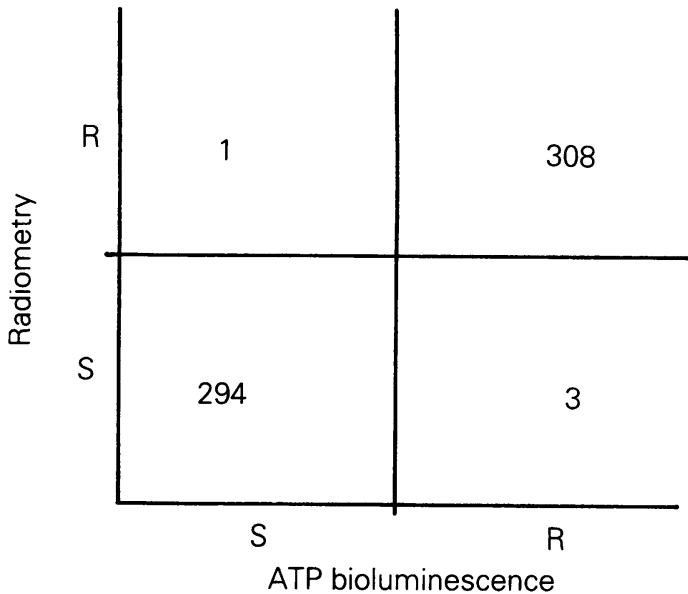

Figure 3 Comparison of 606 susceptibility results (representing 119 strains of mycobacteria) obtained by ATP bioluminescence and radiometry.

simulate resistance. In our experience a McFarland 0.5 standard was equivalent to about $10^{6}$ mycobacteria $/ \mathrm{ml}$. The addition of $0.5 \mathrm{ml}$ of this starting suspension to the tubes containing antimicrobial agents gave a final inoculum of about $10^{5}$ mycobacteria $/ \mathrm{ml}$. Some difficulty in obtaining the correct inoculum was encountered because of the floccular nature of $M$ tuberculosis in particular. This was overcome by the addition of $0.05 \%$ Tween 80 to the suspending medium. The efficient extraction of ATP also depends on the physical condition of the organism. Janasek and colleagues found that the amount of ATP extracted from mycobacterium BCG varied following storage at different temperatures. ${ }^{22}$ Other methods for the extraction of bacterial ATP have been tried, including heating in ethanol, ${ }^{23}$ a combination of heat and chloroform, ${ }^{24}$ and exposure to chemicals such as sulphuric acid, ${ }^{25}$ butanol, ${ }^{26}$ and dimethyl sulphoxide. ${ }^{27}$ Prioli et al compared five different methods of ATP extraction and found that boiling TRIS/EDTA buffer was the best for extracting ATP from mycobacteria. ${ }^{27}$ The ATP method used in this study was based on that described by Nilsson et al. ${ }^{16}$ We found, however, that slight modifications were required before satisfactory results were obtained. These included increasing the amount of mycobacterial culture from 50 to $100 \mu \mathrm{l}$, extending the heat extraction process from 90 seconds to 5 minutes, and reducing the volume of monitoring reagent from 100 to $50 \mu \mathrm{l}$. These measures increased the sensitivity of the method, ensured total killing of the mycobacterial strain, and reduced the cost per test.

The antimicrobial susceptibility methods used in this study are fundamentally different. The proportional method assumes that if $1 \%$ of the mycobacterial population is resistant to a drug then treatment will not be clinically effective. The ATP bioluminescence assay is based on broth dilution, where the potency of a drug is assessed in a more arbitrary manner. Resistance is reflected by an ATP index that is similar to that obtained from drug-free con- 
trols. Susceptibility is shown when the ATP activity remains at a low level during incubation, and falls to $40 \%$ or less of the control reading. This criterion has been widely accepted for rapid susceptibility testing using ATP bioluminescence. ${ }^{17-19}$ Both ATP and radiometric methods were comparable in terms of speed; results were available in seven days, although contamination of ATP readings was a problem simply because all microorganisms contain ATP. A single reading at day 7 of incubation would obviously alleviate this problem.

Beckers et al noted that in most cases of disagreement between ATP and conventional methods, the conventional method showed resistance while the luminescence technique showed susceptibility. ${ }^{17} \mathrm{We}$ concur with these findings, which may be due to the closer contact between bacterium and antimicrobial agent in fluid media. Furthermore, degradation of drugs may occur during the prolonged incubation necessary for conventional susceptibility techniques. Beckers et al also observed that discrepancies with rifampicin and streptomycin were caused by phospholipid and heat inactivation, respectively, if $\mathrm{LJ}$ medium was used. ${ }^{17}$ We used Middlebrook 7H10 agar and encountered no such problems.

Despite these limitations regarding direct comparison of techniques, ATP bioluminescence permits rapid susceptibility testing of mycobacteria. The technique seems to be reliable, can be automated, and requires no radioactive substances. It has considerable potential for use within the clinical microbiology laboratory.

1 Collins CH, Lyne PM. Microbiological methods. 5th edn London: Butterworths, 1984.

2 Vestal AL. Procedures for the isolation and identification of mycobacteria. DHEW Publication No. (CDC) 75-8230. Atlanta: Centers for Disease Control, 1975.

3 Canetti G, Froman S, Grosset J, et al. Mycobacteria: laboratory methods for testing drug sensitivity and resistance. Bull WHO 1963;29:565-78.

4 Kent PT, Kubicka GP. Public health mycobacteriology: a guide for the level III laboratory. Atlanta: US Departmen of Health and Human Services, Public Health Service, Centers for Disease Control, 1986.

5 Fauci AS. Acquired immune deficiency syndrome: epidemiologic, clinical immunologic and therapeutic considerations. Ann Intern Med 1984;100:92-106.

6 Collins FM. Mycobacterial disease, immunosuppression, and the acquired immunodeficiency syndrome. Clin
Microbiol Rev 1989;2:360-77.

7 Gold JWM, Armstrong D. Infectious complications of the acquired immunodeficiency syndrome. Proc NY Acad Sci 1985;347:383-93.

8 Collins FM. M avium-complex infections and development of the acquired immunodeficiency syndrome: casual opportunist or casual cofactor? Int $\mathcal{f}$ Leprosy 1986;54:458-74.

9 Cummings DM, Ristroph D, Camargo EE, Larson SM, Wagner HN. Radiometric detection of the metabolic activity of Mycobacterium tuberculosis. $\boldsymbol{f}$ Nucleic Med 1975;16:1189-91.

10 Middlebrook G, Reggiardo Z, Tigertt WD. Automatable radiometric detection of growth of Mycobacterium radiometric detection of growth of Mycobacterium tuberculosis in se

11 Kertcher JA, Chen MF, Charache $P$, et al. Rapid radiometric susceptibility testing of Mycobacterium tuberculosis. Am Rev Respir Dis 1978;117:631-7.

12 Vincke G, Yegers O, Vanachter H, Jenkins PA, Butzler JP. Rapid susceptibility testing of Mycobacterium tuberculosis by a radiometric technique. $\mathcal{\exists}$ Antimicrob Chemother 1982;10:351-4.

13 Hawkins JE. Rapid mycobacterial susceptibility tests. Clinical Microbiology Newsletter 1986;8:101-4.

14 Tarrand J, Groschel DHM. Evaluation of the BACTEC radiometric method for detection of $1 \%$ resistant popuradiometric method for detection of $1 \%$ resistant popu1985;21:941-6.

15 D'Eustachio AJ, Johnson DR. Adenosine triphosphate content of bacteria. Fed Proc 1968;27:761.

16 Nilsson LE, Hoffner SE, Ansehn S. Rapid susceptibility testing of Mycobacterium tuberculosis by bioluminescence assay of mycobacterial ATP. Antimicrob Agents Chemother 1988;32:1208-12.

17 Beckers B, Lang HRM, Schimke D, Lammers A. Evaluation of a bioluminescence assay for rapid antimicrobial susceptibility testing of mycobacteria. Eur $\mathcal{f}$ Clin Microbiol 1985;4:556-61.

18 Wheat PF, Hastings JGM, Spencer RC. Rapid antibiotic susceptibility tests on Enterobacteriaceae by ATP biosusceptibility tests on Enterobacteriaceae by

19 Wheat PF, Spencer RC, Hastings JGM. A novel luminometer for antimicrobial susceptibility tests on Grampositive cocci by ATP bioluminescence. $\mathcal{f}$ Med Microbiol 1989;29:277-82

20 Middlebrook G, Cohn ML. Bacteriology of tuberculosis: laboratory methods. Am $\mathcal{F}$ Public Health 1958;48: 844-53.

21 Stager CE, Davis JR, Saccomani $M N$, Ortigoza CO, Wende RD, Raleigh JW. Modified inoculum for radiometric susceptibility testing of Mycobacterium tuberculosis. Curr Microbiol 1988;17:243-7.

22 Janasek $W$, Aleksandrowicz J, Sitkiewicz D. The use of the firefly bioluminescent reaction for the rapid detection firefly bioluminescent reaction for the rapid detection and counting

23 John SJB. Determination of ATP in chlorella with luciferin-luciferase enzyme system. Anal Biochem 1970; 37:409-16.

24 Lundin A, Thore A. Comparison of methods for the extraction of bacterial adenine nucleotides determined by firefly assay. Appl Microbiol 1975;30:713-21.

25 Forrest WW, Walker DJ. Synthesis of reverse materials for endogenous metabolism in Streptococcus faecalis. f Bacteriol 1965;89:1448-52.

26 Sharpe AN, Woodrow MN, Jackson AK. Adenosine triphosphate (ATP) levels in foods contaminated by bacteria. $¥$ A ppl Bacteriol 1970;33:758-67.

27 Prioli RP, Tanna A, Brown JN. Rapid methods for counting bacteria-comparison of methods for extraction of mycobacterial adenosine triphosphate (ATP) determined by firefly luciferase assay. Tubercle 1985;66: 99-108. 